\title{
Freqüência de Doença Cardiovascular Aterosclerótica e de seus Fatores de Risco em Pacientes com Lúpus Eritematoso Sistêmico
}

\section{Frequency of Atherosclerotic Cardiovascular Disease and its Risk Factors in Patients with Systemic Lupus Erythematosus}

\author{
Rosa Weiss Telles ${ }^{(1)}$, Cristina Costa Duarte Lanna ${ }^{(2)}$, Gilda Aparecida Ferreira ${ }^{(3)}$, \\ Marco Antonio Parreiras de Carvalho ${ }^{(4)}$, Antonio Luiz Ribeiro ${ }^{(5)}$
}

\section{RESUMO}

Introdução: a patogênese da doença arterial coronariana no lúpus eritematoso sistêmico não está completamente estabelecida, porém fatores relacionados ao lúpus e ao seu tratamento parecem se associar à presença de fatores de risco tradicionais. Esses fatores de risco estão presentes em indivíduos com lúpus em uma freqüência maior em relação à população geral e correlacionam-se à presença de doença cardiovascular manifesta nessa população. Objetivos: determinar a freqüência da doença cardiovascular manifesta e dos fatores de risco em pacientes com lúpus acompanhados no Serviço de Reumatologia do Hospital das Clínicas da UFMG. Métodos: avaliação transversal de 172 pacientes com lúpus. Eventos cardiovasculares, fatores de risco tradicionais e manifestações clínico-laboratoriais do lúpus foram avaliados. Resultados: a média de idade (DP) foi de 38,5 anos (11,2 anos): a maioria do sexo feminino $(95,9 \%)$ e da etnia não-branca $(64,5 \%)$. Identificou-se doença cardiovascular em oito pacientes $(4,7 \%)$, com 11 diagnósticos diferentes. Três apresentaram evento coronariano; três, acidente vascular cerebral isquêmico; e cinco, doença arterial periférica. $\mathrm{O}$ fator de risco mais freqüente foi hipertensão arterial sistêmica $(48,8 \%)$, seguido por dislipidemia em $70(40,7 \%)$ pacientes e hipertrigliceridemia em 51 (29,7\%). O c-LDL $\geq 100 \mathrm{mg} / \mathrm{dl}$ foi encontrado em $77(44,8 \%)$ pacientes. Entre as 165 pacientes do sexo feminino, $67(40,6 \%)$ encontravam-se na pósmenopausa, e $43,3 \%$ tiveram o diagnóstico de menopausa precoce. Conclusões: o presente estudo descreve a freqüência dos fatores de risco para doença arterial coronariana em uma amostra significativa de pacientes brasileiros com lúpus. A importância do reconhecimento de tais fatores encontra-se na possibilidade de modificação e tratamento de alguns deles. O impacto da identificação e do tratamento de fatores de risco em pacientes com lúpus para prevenção de eventos cardiovasculares deve ser objeto de novos estudos.

Palavras-chave: lúpus eritematoso sistêmico, aterosclerose, fatores de risco, doença cardiovascular.

\begin{abstract}
Introduction: the pathogenesis of coronary disease in systemic lupus erythematosus patients is not completely understood. Risk factors associated with lupus or its treatment may be associated with traditional risk factors for coronary disease. Such risk factors are more common in patients with lupus. Objective: to determine the frequency of cardiovascular disease and traditional risk factors in patients with systemic lupus erythematosus at Rheumatology Division of Hospital das Clínicas of Minas Gerais Federal University. Methods: 172 patients with systemic lupus erythematosus were evaluated. Cardiovascular events, traditional risk factors and clinical-laboratorial findings were investigated in this cross-sectional research. Results: the mean age (SD) of the patients was 38.5 years (11.2 years). $95.9 \%$ were female and $64.5 \%$ were non white. Cardiovascular disease was identified in 8 patients (4.7\%), with 11 different diagnoses. Three patients had coronary insufficiency, three had stroke and five had peripheral arterial disease. Systemic arterial hypertension was the most frequent risk factor (48.8\%), followed by dyslipidemia in 70 patients (40.7\%) and hypertriglyceridemia in 51 patients (29.7\%). LDL-c $\geq 100 \mathrm{mg} / \mathrm{dl}$ was found in 77 patients (44.8\%). Among $165 \mathrm{fe}$ male patients, 67 (40.6\%) had menopause, $43.3 \%$ of them with early menopause. Conclusion: the present study describes the frequency of risk factors for coronary artery disease in a Brazilian sample of lupus patients. The impact of recognition and management of those risk factors in prevention of cardiovascular events should be object of others studies.
\end{abstract}

Keywords: systemic lupus erythematosus, atherosclerosis, risk factors for coronary disease, cardiovascular disease.

\footnotetext{
Recebido em 14/02/07. Aprovado, após revisão, em 25/05/07.

Serviços de Reumatologia e de Cardiologia do Hospital das Clínicas da Universidade Federal de Minas Gerais (UFMG), Departamento do Aparelho Locomotor e Departamento de Clínica Médica da Faculdade de Medicina da UFMG - Pós-graduação em Clínica Médica da Faculdade de Medicina da UFMG, Áreas de Concentração em Cardiologia e Reumatologia.

1. Reumatologista titulada pela Sociedade Brasileira de Reumatologia e membro do Projeto Pronuclear da Sociedade Brasileira de Reumatologia

2. Professora adjunta, doutora de Reumatologia do Departamento do Aparelho Locomotor da Faculdade de Medicina da UFMG.

3. Doutora em Reumatologia pelo Departamento de Reumatologia da Universidade Federal de São Paulo (Unifesp).

4. Professor adjunto, doutor de Reumatologia do Departamento do Aparelho Locomotor da Faculdade de Medicina da UFMG.

5. Professor adjunto, doutor de Cardiologia do Departamento de Clínica Médica da Faculdade de Medicina da UFMG.

Endereço para correspondência: Rosa Weiss Telles, Rua Muzambinho, 104/201, CEP 30310-280, Belo Horizonte, MG, Brasil, e-mail: rwtelles@ig.com.br
} 


\section{INTRODUÇÃO}

O lúpus eritematoso sistêmico (LES) é uma doença inflamatória do tecido conjuntivo de etiologia desconhecida. Caracteriza-se clinicamente por períodos de exacerbação e remissão em pacientes que, em sua maioria, são cronicamente enfermos $^{(1)}$. Apresenta-se de forma polimórfica, acometendo diferentes órgãos e sistemas. A sua prevalência na população geral varia de 15 a 50/100.000 habitantes, com predomínio de mulheres, principalmente durante o período reprodutivo.

Os pacientes com LES dispõem hoje de considerável arsenal para tratamento de sua doença, incluindo antiinflamatórios não-hormonais, corticóides, antimaláricos, imunossupressores, imunoglobulina endovenosa, anticorpos anti-CD20, além de transplante de medula óssea. Como conseqüência direta do aumento do conhecimento e das possibilidades de tratamento do LES, a sobrevida desses pacientes tem aumentado consideravelmente nos últimos 40 anos, levando ao aparecimento e reconhecimento de novas causas de morbidade e mortalidade, entre elas a doença aterosclerótica.

A doença arterial coronariana (DAC) aterosclerótica é uma importante causa de morbidade e mortalidade em pacientes com LES. Eventos cardiovasculares e cerebrovasculares ocorrem com maior freqüência em pacientes com lúpus quando comparados à população geral, especialmente em mulheres na pré-menopausa ${ }^{(2-4)}$. A taxa de mortalidade secundária à doença aterosclerótica no lúpus varia entre $6 \%$ e $16 \%{ }^{(5)}$ dos casos, podendo chegar a $30 \%^{(6)}$. A freqüência de DAC em várias coortes foi determinada entre $4,9 \% \mathrm{e}$ $10 \%^{(4,6-9)}$. Manzi et al..$^{(4)}$ calcularam que o risco de uma paciente com LES, entre 35 e 44 anos de idade, apresentar infarto agudo do miocárdio é aproximadamente 50 vezes maior do que o risco de uma mulher da população geral na mesma faixa.

A patogênese da DAC no LES não está completamente estabelecida, porém fatores relacionados ao LES e ao seu tratamento parecem se associar à presença de fatores de risco tradicionais para doença arterial coronariana. Os fatores de risco tradicionais, apesar de não serem os únicos responsáveis pelo desenvolvimento da aterosclerose nesses pacientes, estão presentes em indivíduos com lúpus com maior freqüência em relação à população geral ${ }^{(10)}$ e associados à presença de doença cardiovascular manifesta no LES $^{(2,11,12)}$.

Apesar do conhecimento cada vez maior da importância da investigação e do controle de fatores de risco modifi- cáveis nas prevenções primária e secundária de DAC, o reconhecimento desses fatores por médicos e pacientes é falho. Além disso, o tratamento da dislipidemia ainda não é realizado de forma ideal ${ }^{(13-15)}$. Por outro lado, populações de alto risco para DAC, como pacientes com história pregressa de eventos cardiovasculares ateroscleróticos, diabéticos e indivíduos com LES, devem ter controle mais rigoroso desses fatores de risco ${ }^{(16,17)}$.

Os objetivos do presente estudo foram determinar a freqüência da doença cardiovascular (DCV) manifesta e dos fatores de risco tradicionais para doença arterial coronariana em pacientes com LES acompanhados no Serviço de Reumatologia do Hospital das Clínicas da UFMG.

\section{PACIENTES E MÉTODOS}

Trata-se de um estudo clínico transversal realizado no Serviço de Reumatologia do Hospital das Clínicas da Universidade Federal de Minas Gerais no período de maio de 2005 a fevereiro de 2006. Os critérios de inclusão utilizados para o estudo foram: diagnóstico de LES, segundo os critérios de classificação de 1982 (revisados em 1997)(18,19) do Colégio Americano de Reumatologia; idade igual ou superior a 18 anos e assinatura do termo de consentimento livre e esclarecido após informação.

Foram incluídos, consecutivamente, 172 pacientes ambulatoriais, entre os 300 acompanhados no Serviço. A comparação entre os pacientes que participaram do estudo $(\mathrm{n}=172)$ e aqueles que não participaram $(\mathrm{n}=128)$ não mostrou diferença significativa quanto a idade, idade ao diagnóstico do LES, etnia e tempo de escolaridade. As manifestações clínicas e laboratoriais presentes nos critérios de classificação para LES também não mostraram diferença significativa, a não ser maior freqüência de sexo masculino $(\mathrm{p}=0,043)$, ausência de FAN $(\mathrm{p}=0,020)$ e positividade do anticorpo anticardiolipina $(\mathrm{p}=0,042)$ (no grupo de pacientes que não participou do estudo).

O protocolo específico do estudo foi preenchido por um dos autores (RWT) após avaliação dos pacientes no dia agendado para consulta. Os prontuários foram revisados em busca de registros de DCV e de fatores de risco para DAC, bem como de manifestações clínico-laboratoriais e de tratamento do LES ${ }^{(18-20)}$. Foram pesquisadas ainda as manifestações da síndrome do anticorpo antifosfolípide ${ }^{(21)}$ e mensurado o índice de dano, segundo proposição do SLICC/ACR (Systemic Lupus International Collaborating Clinics/American College of Rheumatology) $)^{(22)}$. 
As definições de doença cardiovascular e dos fatores de risco para doença arterial coronariana encontram-se no Quadro $1^{(6,16,23-27)}$.

A hipertensão arterial sistêmica (HAS), o diabetes melito (DM) e a dislipidemia foram classificados como pregressos, quando presentes por pelo menos um ano, porém ausentes no momento do estudo, ou atuais, quando presentes no momento da coleta dos dados.

Para obtenção do índice tornozelo-braquial foi utilizada a razão da pressão sistólica do tornozelo sobre a pressão sistólica braquial medida pelo Doppler.

Considerando-se as recomendações atuais para controle da dislipidemia em pacientes de alto risco para eventos cardiovasculares, inclusive em pacientes com LES ${ }^{(16,17)}$, os pacientes também foram divididos baseando-se em valores de $\mathrm{c}-\mathrm{LDL} \geq 100 \mathrm{mg} / \mathrm{dl}$.

$\mathrm{O}$ risco de evento coronariano em determinado indivíduo está associado à combinação dos fatores de risco descritos. Foram utilizadas três formas de agrupamento de fatores de risco no presente estudo: número total de fatores de risco para doença coronariana, escore de Framingham e síndrome metabólica:

- Número total de fatores de risco para doença coronariana: idade e sexo (homens com idade igual ou superior a 45 anos e mulheres com idade igual ou superior a 55 anos), história familiar positiva de doença arterial coronariana precoce em parentes de primeiro grau (HF), HAS, tabagismo, DM, dislipidemia e hipertrigliceridemia ${ }^{(16)}$.
- Escore de Framingham (EF): as variáveis utilizadas para construção do escore de Framingham foram: idade e sexo, pressões arteriais sistólica e diastólica, c-LDL, c-HDL, tabagismo e $\mathrm{DM}^{(28)}$. A partir da soma de pontos fornecida por cada variável, é estimado o risco de evento cardiovascular em dez $\operatorname{anos}^{(16)}$. Risco $\geq 20 \%$ representa alto risco para evento; $10 \%-20 \%$, risco intermediário; e $<10 \%$, risco baixo.

- Síndrome metabólica: para diagnóstico da síndrome devem estar presentes pelo menos três dos cinco itens obesidade abdominal, hipertrigliceridemia, baixos níveis de c-HDL $(<40 \mathrm{mg} / \mathrm{dl}$ para homens e $<50 \mathrm{mg} / \mathrm{dl}$ para mulheres), pressão arterial sistólica $\geq 135 \mathrm{mmHg}$ ou pressão arterial diastólica $\geq 85 \mathrm{mmHg}$ e resistência à insulina

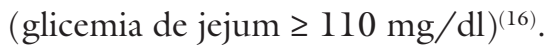

\section{ANÁLISE ESTATÍSTICA}

Um banco de dados foi construído utilizando-se o programa EpiData v3.0 (EpiData Association, Odense, Denmark). A entrada dos dados no banco foi verificada por meio do recurso de dupla entrada disponível no programa. A análise estatística foi realizada usando-se o pacote estatístico SPSS/Windows v12.0 (SPSS Inc., Chicago, IL USA.). Variáveis categóricas foram descritas como proporção e variáveis contínuas por média e desvio-padrão (DP) ou mediana e intervalo interquartil (IIq), quando apropriado.

Este trabalho foi aprovado pelo Comitê de Ética em Pesquisa em Seres Humanos da Universidade Federal de Minas Gerais, em maio de 2005.

QUADRO 1

DEFINIÇÃO DE FATORES DE RISCO PARA DOENÇA ARTERIAL CORONARIANA E DE EVENTOS CARDIOVASCULARES ATEROSCLERÓTICOS

\begin{tabular}{ll}
\hline Fator de risco & Definições \\
\hline Evento coronariano prévio & Relato de história de IAM, angioplastia ou CRVM \\
\hline AVCI & História de AVC com déficit focal com duração maior do que 24 horas ou seqüela ao exame físico \\
\hline Doença arterial periférica & $\begin{array}{l}\text { Queixa de claudicação intermitente ou déficit de pulso distal de membros inferiores, associada a índice tornozelo-braquial me- } \\
\text { nor do que } 0,9 \text { ao Doppler; ou amputação de extremidades, cirurgia de bypass e arteriografia demonstrando obstrução arterial }\end{array}$ \\
\hline História familiar positiva para DAC & $\begin{array}{l}\text { História de IAM, angioplastia, CRVM ou morte súbita cardíaca em parentes de primeiro grau (idade inferior a } 55 \text { anos em } \\
\text { homens e } 65 \text { anos em mulheres) }\end{array}$ \\
\hline Hipertensão arterial sistêmica & PAS $\geq 140 \mathrm{mmHg}$ ou PAD $\geq 90 \mathrm{mmHg}$ em pelo menos duas ocasiões ou uso de anti-hipertensivo \\
\hline Tabagismo & Uso de qualquer quantidade de tabaco no último mês \\
\hline Obesidade & Índice de massa corporal acima de $30 \mathrm{~kg} / \mathrm{m}^{2}$ \\
\hline Obesidade abdominal & Circunferência abdominal, medida na crista ilíaca, acima de $88 \mathrm{~cm}$ em mulheres e $102 \mathrm{~cm}$ em homens \\
Diabetes melito & Glicemia de jejum $\geq 126 \mathrm{mg} / \mathrm{dl}$ em, pelo menos, duas ocasiões, ou uso de hipoglicemiantes orais ou insulina \\
\hline Dislipidemia & ColT $\geq 200 \mathrm{mg} / \mathrm{dl}$ ou c-HDL < $40 \mathrm{mg} / \mathrm{dl}$ ou c-LDL $\geq 130 \mathrm{mg} / \mathrm{dl}$ ou uso de hipolipemiantes orais \\
\hline Hipertrigliceridemia & TGLs $\geq 150 \mathrm{mg} / \mathrm{dl}$ \\
\hline Menopausa & Última menstruação espontânea há mais de um ano; ou uso de TRH; ou irregularidade menstrual ou amenorréia há menos de \\
\hline
\end{tabular}

$\mathrm{DAC}=$ doença arterial coronariana; IAM = infarto agudo do miocárdio; $C R V M=$ cirurgia de revascularização miocárdica; $A V C I=$ acidente vascular cerebral isquêmico; PAS = pressão arterial sistêmica; $\mathrm{PAD}=$ pressão arterial diastólica; ColT = colesterol total; $\mathrm{c}-\mathrm{HDL}=$ colesterol de alta densidade; $\mathrm{c}-\mathrm{LDL}=\mathrm{colesterol}$ de baixa densidade; $\mathrm{TGLS}=$ triglicérides; TRH = terapia de reposição hormonal; FSH = hormônio folículo estimulante. 


\section{RESULTADOS}

Foram incluídos 172 pacientes no estudo. A média de idade (DP) foi de 38,5 anos (11,2 anos). A maioria era do sexo feminino $(95,9 \%)$ e da etnia não-branca $(64,5 \%)$. A mediana (IIq) do tempo de doença foi de 99,0 meses (54,5-154,0 meses) e a do tempo de acompanhamento foi de 84,0 meses (53,5-133,5 meses). A média de idade (DP) ao diagnóstico do LES foi de 29,1 anos (10,0 anos), com mediana de 27,0 anos, idade mínima de 11,0 anos e máxima de 61,0 anos.

As características clínico-laboratoriais dos pacientes encontram-se descritas na Tabela 1 . Nenhum deles apresentou doença renal em diálise. A síndrome do anticorpo antifosfolípide foi diagnosticada em nove pacientes, quatro dos quais apresentavam história de morbidade gestacional e seis, de trombose vascular. História de trombose venosa profunda foi identificada em cinco indivíduos e trombose arterial, em um. A média (DP) do índice de dano foi 1,74 (1,65), com mediana (IIq) igual a 1 (0-3), mínimo de 0 e máximo de 7.

\section{TABELA 1}

Características CLÍNICO-LABORATORIAIS DE 172 PACIENTES COM LÚPUS ERITEMATOSO SISTÊMICO

\begin{tabular}{lc}
\hline Características clínico-laboratoriais* & $\begin{array}{c}\text { Pacientes com LES (N = 172) } \\
\text { N }(\%)\end{array}$ \\
\hline Manifestações mucocutâneas** & $139(80,8)$ \\
\hline Serosite (pleurite ou pericardite) & $54(31,4)$ \\
\hline Artrite & $111(64,5)$ \\
\hline Manifestações neuropsiquiátricas*** & $26(15,1)$ \\
\hline Alterações hematológicas & $151(87,8)$ \\
\hline Leucopenia/linfopenia & $148(98)$ \\
\hline Plaquetopenia & $25(16,6)$ \\
\hline Anemia hemolítica & $27(17,9)$ \\
\hline Vasculite cutânea & $58(33,7)$ \\
\hline Nefrite & $105(61)$ \\
\hline Proteinúria $\geq 3,5$ g/24 horas & $41(39)$ \\
\hline FAN & $172(100)$ \\
\hline Anti-Sm positivo ${ }^{\dagger}$ & $46(27,2)$ \\
\hline Anti-DNA nativo positivo ${ }^{\ddagger}$ & $80(46,8)$ \\
\hline VDRL positivo ${ }^{\ddagger}$ & $13(7,6)$ \\
\hline LA positivo & $39(23,1)$ \\
\hline aCL positiva ${ }^{\dagger}$ & $23(13,4)$ \\
\hline
\end{tabular}

FAN = fator antinúcleo; anti-Sm = anticorpo anti-Smith; anti-DNA nativo = anticorpo antiácido desoxirribonucléico nativo; $\mathrm{VDRL}=$ teste do veneral disease research laboratory; $\mathrm{LA}=$ anticoagulante lúpico; $\mathrm{aCL}$ = anticardiolipina IgG ou IgM.

*Definidas segundo os critérios para classificação do LES/ACR, a não ser quando indicado.

**Incluem: eritema malar, fotossensibilidade, úlceras orais, lúpus discóide e lesão subaguda do LES.

*** Incluem: convulsão, psicose, mielite transversa, acidente vascular cerebral ou alteração de par craniano.

\#Definida como: proteinúria superior a $0,5 \mathrm{~g} / 24 \mathrm{~h}$ ou $\geq 3+$ em exame de urina de rotina, cilindros celulares (eritrocitários, hemoglobínicos, granulares, tubulares ou mistos), biópsia renal compatível com nefrite lúpica ou doença renal terminal (com ou sem transplante) atribuída à nefrite lúpica.

†Não pesquisado(a) em três pacientes.

$\ddagger$ Não pesquisado em um paciente.
Em relação aos medicamentos indicados para tratamento do LES durante o acompanhamento da doença, 98,8\% dos pacientes usou corticóide, 89,5\%, antimaláricos e 69,8\%, imunossupressores. A ciclofosfamida foi o imunossupressor mais usado (89 pacientes), seguido pela azatioprina (81 pacientes). Outros imunossupressores como metotrexato, ciclosporina e micofenolato mofetil foram usados por 30 pacientes. No momento da coleta de dados, o corticóide ainda era a medicação usada pela maioria dos pacientes $(79,1 \%)$, seguido pelos antimaláricos $(51,2 \%)$ - 95\% deles usavam difosfato de cloroquina. Setenta e oito pacientes (45,3\%) usavam imunossupressores: azatioprina em 26 ciclofosfamida em 23 e outros em 19 pacientes.

A freqüência dos fatores de risco está apresentada na Tabela 2. O fator de risco mais freqüente foi HAS, presente em 48,8\% dos indivíduos estudados. Dislipidemia ocorreu em $70(40,7 \%)$ pacientes, sendo o nível baixo de c-HDL a principal alteração de perfil lipídico, presente em 47 deles. Hipertrigliceridemia ocorreu em 51 (29,7\%) pacientes. Observou-se que $77(44,8 \%)$ pacientes apresentavam c-LDL $\geq 100 \mathrm{mg} / \mathrm{dl}$. A obesidade e a obesidade abdominal estavam presentes em $36(20,9 \%)$ e 75 (43,3\%) doentes, respectivamente. História pregressa de tabagismo foi relatada por 54 pacientes $(31,4 \%)$. Destes, $23(13,4 \%)$ relatavam tal hábito no momento da coleta de dados. HAS em $17(9,9 \% \%)$ pacientes, dislipidemia em $16(9,3 \%)$ e DM em três $(1,7 \%)$ foram transitórios, sendo classificados como pregressos.

Entre as 165 pacientes do sexo feminino, 67 (40,6\%) encontravam-se na pós-menopausa. Destas, 29 (43,3\%) preenchiam critério para menopausa precoce.

Quanto ao número total de fatores de risco, 45 pacientes $(26,2 \%)$ não apresentaram nenhum e $39(22,7 \%)$ tinham três ou mais. Pelo menos um fator de risco estava presente em 50 indivíduos $(29,1 \%)$ e $38(22,1 \%)$ tinham dois. A síndrome metabólica foi diagnosticada em 46 (28,4\%) dos 162 pacientes sem DM no momento do estudo (Tabela 2). $\mathrm{O}$ escore de Framingham calculado para os pacientes do estudo encontra-se representado na Gráfico 1. Dos 172 pacientes avaliados $163(94,7 \%)$ tinham baixo risco de evento nos dez anos seguintes, sete $(4,1 \%)$ apresentavam risco intermediário e dois $(1,2 \%)$ pacientes tinham risco alto.

A DCV manifesta foi identificada em oito pacientes $(4,7 \%)$, todos do sexo feminino, com 11 diagnósticos diferentes. Três deles apresentaram evento coronariano prévio $(1,7 \%)$, três tiveram acidente vascular cerebral isquêmico (AVCi) (1,7\%) e cinco, doença arterial periférica (2,9\%). A doença arterial periférica ocorreu isoladamente em três pacientes - em combinação com evento coronariano em um, 
TABELA 2

FATORES DE RISCO TRADICIONAIS PARA DOENÇA ARTERIAL CORONARIANA EM 172 PACIENTES COM LÚPUS ERITEMATOSO SISTÊMICO

\begin{tabular}{|c|c|}
\hline Fatores de risco tradicionais para DAC & Pacientes com LES \\
\hline $\operatorname{HAS}[n(\%)]$ & $84(48,8)$ \\
\hline PAS mmHg mediana (IIq) & $120(110-130)$ \\
\hline PAD mmHg mediana (IIq) & $80(70-83)$ \\
\hline Obesidade abdominal [n (\%)] & $75(43,6)$ \\
\hline Circunferência abdominal cm mediana (IIq) & $87,5(79,8-96,0)$ \\
\hline Dislipidemia [n (\%)] & $70(40,7)$ \\
\hline Colesterol total $\geq 200 \mathrm{mg} / \mathrm{dl}[\mathrm{n}(\%)]$ & $34(19,7)$ \\
\hline Colesterol total $\mathrm{mg} / \mathrm{dl}$ mediana (IIq) & $165,5(145,5-191,5)$ \\
\hline $\mathrm{c}-\mathrm{LDL} \geq 130 \mathrm{mg} / \mathrm{dl}[\mathrm{n}(\%)]$ & $31(18,0)$ \\
\hline c-LDL mg/dl mediana (IIq) & $96(75-115,5)$ \\
\hline $\mathrm{c}-\mathrm{HDL}<40 \mathrm{mg} / \mathrm{dl}[\mathrm{n}(\%)]$ & $47(27,3)$ \\
\hline c-HDL mgdl mediana (IIq) & $46(39-56,5)$ \\
\hline $\mathrm{TGL} \geq 150 \mathrm{mg} / \mathrm{dl}[\mathrm{n}(\%)]$ & $51(29,7)$ \\
\hline TGL mg/dl mediana (IIq) & $115,5(81,5-160)$ \\
\hline$c-L D L \geq 100 \mathrm{mg} / \mathrm{dl}[\mathrm{n}(\%)]$ & $77(44,8)$ \\
\hline Menopausa** $[\mathrm{n}(\%)]$ & $67(40,6)$ \\
\hline Menopausa precoce ${ }^{* \star *}[\mathrm{n}(\%)]$ & $29(43,3)$ \\
\hline Tabagismo atual $[\mathrm{n}(\%)]$ & $23(13,4)$ \\
\hline Obesidade $[\mathrm{n}(\%)]$ & $36(20,9)$ \\
\hline IMC kgm² mediana (IIq) & $25,6(23,0-29,0)$ \\
\hline História familiar* [n (\%)] & $24(14)$ \\
\hline $\mathrm{DM}[\mathrm{n}(\%)]$ & $10(5,8)$ \\
\hline Glicemia mg/dl mediana (IIq) & $78(72-84,5)$ \\
\hline Síndrome metabólica***** $[\mathrm{n}(\%)]$ & $46(28,4)$ \\
\hline
\end{tabular}

$\mathrm{DAC}=$ doença arterial coronariana; $\mathrm{DM}=$ diabetes melito; $\mathrm{HAS}=$ hipertensão arterial sistêmica; $c-L D L=$ colesterol de baixa densidade; $c-H D L=$ colesterol de alta densidade; $\mathrm{TGL}=$ triglicérides; $\mathrm{PAS}$ = pressão arterial sistólica; $\mathrm{PAD}$ = pressão arterial diastólica; $\mathrm{c}-\mathrm{LDL}=$ colesterol de baixa densidade; $\mathrm{c}-\mathrm{HDL}=$ colesterol de alta densidade; $\mathrm{TGL}=$ triglicérides; IMC = índice de massa corporal; IIq = intervalo interquartil.

*Dois pacientes não informaram sobre história familiar.

**165 pacientes do sexo feminino.

*** Dentre as 67 pacientes com menopausa

****Em 162 pacientes (sem DM atual).

\section{GRÁFICO 1}

ESCORE DE RISCO DE EVENTO CORONARIANO EM DEZ ANOS (FRAMINGHAM)

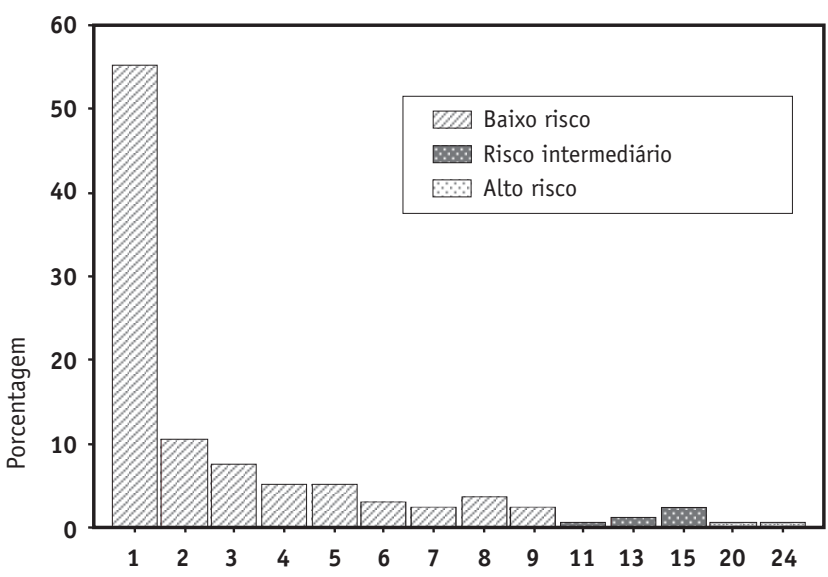

Escore de risco de Framingham e com evento coronariano e AVCi em outro. Dois pacientes apresentaram AVCi isolado, e evento coronariano prévio ocorreu isoladamente em um. O diagnóstico de doença arterial periférica foi realizado após constatação de déficit de pulso ao exame físico e realização de Doppler em quatro pacientes assintomáticas. $\mathrm{O}$ quinto diagnóstico foi realizado em indivíduo com história de gangrena seca em artelhos e realização de arteriografia de membros inferiores.

Somente uma paciente com evento cardiovascular preenchia os critérios para síndrome do anticorpo antifosfolípide (paciente número 3 na Tabela 3 ). Teste positivo para pesquisa de anticoagulante lúpico foi identificado em outra paciente, porém uma única vez (paciente número 2 na Tabela 3). Nessa paciente, o anticoagulante lúpico positivo foi identificado sete meses após o evento coronariano, com título do anticorpo anticardiolipina IgM igual a 22,4 MPL e IgG negativo. Testes posteriores para anticoagulante lúpico e anticardiolipina foram negativos. Outra paciente (número 8 na Tabela 3 ) apresentou título de anticardiolipina IgG igual a 24 GPL e IgM negativo e testes para anticoagulante lúpico negativos concomitantes ao diagnóstico de doença arterial periférica por ausência de pulso tibial posterior

TABELA 3

CARACTERÍSTICAS DEMOGRÁFICAS E CLÍNICAS DE OITO PACIENTES DO SEXO FEMININO COM DCV MANIFESTA

\begin{tabular}{|c|c|c|c|c|c|}
\hline Paciente & DCV & $\begin{array}{l}\text { Idade* } \\
\text { (anos) }\end{array}$ & $\begin{array}{c}\text { Tempo } \\
\text { de LES** } \\
\text { (anos) }\end{array}$ & $\operatorname{Risco}^{\dagger}$ & $\begin{array}{l}\text { Fatores } \\
\text { de risco }\end{array}$ \\
\hline 1 & $\begin{array}{l}\text { ECo } \\
\text { DAP }\end{array}$ & $\begin{array}{l}56 \\
60\end{array}$ & $\begin{array}{c}9 \\
13\end{array}$ & $15 \%$ & $\begin{array}{l}\text { HF, Tabag, } \\
\text { Menop }\end{array}$ \\
\hline 2 & ECo & 44 & 19 & $15 \%$ & $\begin{array}{l}\text { HAS, Tabag, } \\
\text { DM, Menop }\end{array}$ \\
\hline 3 & $\begin{array}{l}\text { ECo } \\
\text { DAP } \\
\text { AVCi }\end{array}$ & $\begin{array}{l}45 \\
53 \\
54\end{array}$ & $\begin{array}{l}16 \\
24 \\
25\end{array}$ & $3 \%$ & HF, Disl \\
\hline 4 & $\mathrm{AVCi}$ & 59 & 14 & $6 \%$ & Tabag, Menop \\
\hline 5 & $\mathrm{AVCi} \mathrm{i}^{* * *}$ & 31 & - & $1 \%$ & - \\
\hline 6 & DAP & 66 & 5 & $15 \%$ & Disl, Menop \\
\hline 7 & DAP & 57 & 8 & $20 \%$ & $\begin{array}{l}\text { HAS, DM, } \\
\text { Disl, Menop }\end{array}$ \\
\hline 8 & DAP & 44 & 18 & $1 \%$ & Disl, Menop \\
\hline
\end{tabular}

$\mathrm{DCV}=$ doença cardiovascular; $\mathrm{ECo}=$ evento coronariano; $\mathrm{AVCi}=$ acidente vascular cerebral isquêmico; DAP = doença arterial periférica; HAS = hipertensão arterial sistêmica; $\mathrm{HF}$ = história familiar positiva; Tabag = tabagismo; $\mathrm{DM}$ = diabetes melito; Menop $=$ menopausa; Disl $=$ dislipidemia .

*Idade ao diagnóstico da DCV.

**Tempo de doença até o diagnóstico do evento descrito.

tRisco de evento coronariano em dez anos segundo escore de Framingham (sem considerar o evento ocorrido/diagnosticado).

***Diagnóstico do AVCi concomitante ao início das manifestações do LES, sem fatores de risco associados. 
esquerdo e índice tornozelo-braquial alterado. A Tabela 3 apresenta o escore de Framingham das pacientes com eventos cardiovasculares e resume os principais fatores de risco presentes no momento do diagnóstico do evento coronariano e do AVCi, e no momento do estudo, quando foi diagnosticada a doença arterial periférica.

A baixa freqüência de eventos cardiovasculares ateroscleróticos presentes nos pacientes analisados tornou inadequada a análise estatística da associação entre fatores de risco e DCV.

\section{DISCUSSÃO}

O presente estudo teve como finalidade identificar a presença de fatores de risco tradicionais para doença arterial coronariana, alguns deles modificáveis, em uma população relativamente jovem de pacientes brasileiros com LES. A freqüência de fatores de risco e, principalmente, o papel destes no desenvolvimento da aterosclerose e da DCV em indivíduos com LES continuam sendo objeto de interesse e estudo. No entanto, a definição dos fatores de risco varia de acordo com os autores, prejudicando a interpretação dos nossos dados e a sua comparação com outros já publicados.

A freqüência dos fatores de risco em pacientes com LES parece aumentar ao longo do tempo da doença. Em uma coorte multicêntrica de 852 pacientes seguidos pelo Systemic Lupus International Collaborating Clinics (SLICC), com tempo médio de diagnóstico igual a 5,4 meses no momento da inclusão na coorte e idade média de 34,2 anos, a freqüência de fatores de risco em 232 pacientes aumentou nos primeiros três anos de acompanhamento. As taxas de obesidade abdominal (medida pela razão cintura-quadril), menopausa e hipercolesterolemia aumentaram 87\%, 70,6\% e $63,9 \%$, respectivamente, durante esses três anos de seguimento. HAS estava presente em $56,9 \%$ dos pacientes; menopausa, em 22,5\%; tabagismo atual, em 20,3\%; e DM, em $5,2 \%$ ao final dos três anos ${ }^{(15,29)}$. Em comparação aos indivíduos aqui apresentados, o DM apresentou freqüência semelhante $(5,8 \%)$, e tanto o tabagismo atual quanto a HAS foram mais freqüentes nos pacientes acompanhados pelo SLICC. A presente pesquisa, por ser transversal, não avaliou a frequiência de fatores de risco ao longo do tempo.

A HAS é um fator de risco reconhecido para evento vascular aterosclerótico no lúpus. Rahman et al. ${ }^{(11)}$ descreveram a importante associação entre HAS, hipercolesterolemia e eventos vasculares em pacientes com LES. No estudo de Petri et al. ${ }^{(6)}$, a razão de chance da associação (intervalo de confiança a 95\%) entre eventos cardiovasculares e HAS foi de 3,5 (1,3-9,6). A HAS é mais comum em pacientes com LES do que na população geral ${ }^{(10)}$. Vários fatores podem ser responsáveis por esse achado, como, por exemplo, o uso de corticóides e a síndrome nefrótica secundária à nefrite lúpica. Nessa série, a HAS foi identificada em $48,8 \%$ dos indivíduos com LES, sendo o fator de risco mais freqüente. De forma semelhante, em um estudo com 90 mulheres com LES realizado na Escola Paulista de Medicina-Unifesp, a HAS ocorreu em 44\% delas, com média de idade de 38 anos, sendo também o fator de risco mais freqüente ${ }^{(30)}$. Em contrapartida à freqüência aqui relatada em indivíduos com lúpus, um estudo populacional realizado no projeto Bambuí, em Minas Gerais, identificou HAS em apenas 16\% das mulheres de uma amostra probabilística com idade entre 30 e $59 \operatorname{anos}^{(31)}$

A obesidade, medida pelo IMC, e a obesidade abdominal têm sido descritas em indivíduos com LES, utilizando-se diferentes definições nos diversos trabalhos. Um estudo brasileiro identificou obesidade, definida também como IMC > 30, em 19,5\% de pacientes com LES com média de idade de $34 \operatorname{anos}^{(9)}$, freqüência semelhante à relatada na presente pesquisa $(20,9 \%)$. A obesidade abdominal faz parte da síndrome metabólica, caracterizada pela resistência periférica à insulina e associada ao risco de $\mathrm{DAC}^{(16)}$. No estudo de Avalos et al. ${ }^{(32)}$, a freqüência da resistência à insulina, avaliada pelo índice HOMA (Homeostasis model assessment) [glicemia jejum $(\mathrm{mmol} / \mathrm{l}) \mathrm{x}$ insulina jejum $(\mu \mathrm{U} / \mathrm{ml}) / 22,5]$, foi maior em pacientes com LES, quando comparados a controles. $\mathrm{O}$ aumento da resistência à insulina associou-se a outros fatores de risco, como IMC, nível de lipoproteína (a), triglicérides e baixo nível de colesterol de alta densidade (c-HDL). A síndrome metabólica foi relatada em $27,2 \%$ e 18,6\% dos indivíduos com LES em dois estudos envolvendo 232 e 263 pacientes, respectivamente ${ }^{(33,34)}$. No presente estudo, $28,4 \%$ dos pacientes preencheram critérios para síndrome metabólica, freqüência maior do que as relatadas anteriormente.

A dislipidemia, especialmente os valores de c-LDL alto e c-HDL baixo, está implicada na aterogênese. Alterações no perfil lipídico são encontradas em pacientes com LES mesmo antes do início do tratamento com corticosteróides e de forma dependente da atividade da doença ${ }^{(35)}$. Na população aqui estudada, a dislipidemia foi o terceiro fator de risco mais freqüente, caracterizada principalmente pelos baixos níveis de c-HDL. Em relação ao c-LDL, 18\% dos pacientes apresentavam valores $\geq 130 \mathrm{mg} / \mathrm{dl} \mathrm{e} 44,8 \%$, c-LDL $\geq 100 \mathrm{mg} / \mathrm{dl}$. O nível de c-LDL a ser atingido pelo tratamento da dislipidemia varia de acordo com o risco de evento cardiovascular de cada indivíduo ${ }^{(16)}$. Em pacientes 
com LES, que apresentam alto risco de DCV semelhantemente a pacientes com diabetes melito tipo 2, Wajed et al. ${ }^{(17)}$ propõe que o c-LDL deveria ser mantido abaixo de 100 $\mathrm{mg} / \mathrm{dl}$. Segundo tais recomendações, $18 \%$ dos pacientes estudados deveriam estar em uso de estatinas por possuírem $\mathrm{c}-\mathrm{LDL} \geq 130 \mathrm{mg} / \mathrm{dl}$ e $44,8 \%$ deveriam ser encorajados a mudanças de hábito de vida e seriam usuários potenciais de estatina por apresentarem $\mathrm{c}-\mathrm{LDL} \geq 100 \mathrm{mg} / \mathrm{dl}$. No entanto, apenas seis pacientes $(3,5 \%)$ estavam em uso de estatina no momento do estudo. $\mathrm{O}$ tratamento inadequado da dislipidemia também foi encontrado nos doentes com LES avaliados por Urowitz et al. ${ }^{(15)} \mathrm{e}$ Costenbader et al. ${ }^{(14)}$. Possíveis causas para esse fato são a preocupação médica com outros aspectos considerados mais importantes no tratamento do lúpus, a dificuldade financeira dos pacientes para aquisição de medicamentos e a grande quantidade e variedade de medicamentos usados pelos indivíduos com lúpus, acabando-se por priorizar o uso de certos medicamentos em detrimento de outros.

No LES, o uso de imunossupressores, especialmente a ciclofosfamida, está associado ao aparecimento da falência ovariana. A menopausa tem sido implicada na perda da proteção relativa que mulheres em idade fértil têm em relação aos homens, no que se refere à DCV. A menopausa foi o quarto fator de risco mais freqüente no estudo, estando presente em $40,6 \%$ das mulheres e, de forma precoce, em $43,3 \%$ dessas. O aparecimento da menopausa precoce nessas pacientes deve-se, provavelmente, ao uso de ciclofosfamida, o imunossupressor utilizado por $51,7 \%$ dos indivíduos estudados.

Como alguns fatores de risco tradicionais estão associados ao tratamento ou às manifestações do LES, eles podem ser transitórios. A HAS pregressa ocorreu em 17 $(9,9 \%)$ pacientes no estudo; a dislipidemia pregressa, em $16(9,3 \%)$; e o DM pregresso, em três ( $1,7 \%)$. O papel da presença de fatores de risco transitórios na aterogênese não está definido. Parece que, pelo menos em relação à dislipidemia, a ocorrência de evento coronariano foi semelhante em pacientes com hipercolesterolemia variável e sem hipercolesterolemia, enquanto a presença de hipercolesterolemia sustentada em período de três anos esteve associada à maior freqüência de eventos ${ }^{(36)}$. Permanece a dúvida de quando iniciar o tratamento de fatores de risco que são identificados durante períodos de atividade do lúpus, como episódios de nefrite grave, e que podem, dessa forma, desaparecer após o controle da doença e a diminuição da dose de corticóide.

$\mathrm{O}$ risco de evento coronariano nos próximos dez anos na população geral pode ser estimado utilizando-se o escore de Framingham ${ }^{(16,28)}$. No entanto, em indivíduos com lúpus, o valor do cálculo do escore de Framingham tem sido questionado e é inapropriado para predizer risco de evento coronariano adequadamente ${ }^{(3,4)}$. Isso se deve, provavelmente, ao papel importante que fatores relacionados ao próprio lúpus ou ao seu tratamento representam, em associação com os fatores de risco tradicionais, na aterogênese desses pacientes. $\mathrm{Na}$ amostra aqui avaliada, 1,2\% dos indivíduos irá encontrar-se na categoria de alto risco para evento coronariano nos próximos dez anos, e o acompanhamento prospectivo desses pacientes permitirá a avaliação do papel do escore de Framingham nessa população.

No presente estudo, foi observada uma baixa freqüência de eventos vasculares em relação a outras séries da literatura $^{(6,7,12,14)}$. Essa comparação é dificultada pela inclusão, nos vários estudos, de diferentes diagnósticos, como infarto agudo do miocárdio, angina pectoris, acidente vascular cerebral, morte súbita e ataque isquêmico transitório. Apesar disso, a freqüência de evento coronariano isoladamente foi menor no presente trabalho $(1,7 \%)$ do que a relatada em outras publicações $(3,6 \% \text { a } 7,0 \%)^{(5-7)}$. Essa diferença pode estar relacionada à menor idade média de diagnóstico na presente série $(29,1$ anos), em comparação à idade média de 43 anos relatada por Gladman e Urowitz ${ }^{(7)}$, e ao menor tempo de doença, 8,3 anos no estudo atual, quando comparado a 13,9 anos no estudo de Sultan et al. relatado por Aranow e Ginzler ${ }^{(5)}$. Tanto a idade média ao diagnóstico do LES quanto o tempo de doença são fatores associados ao risco de ocorrência de eventos vasculares ${ }^{(6)}$.

\section{CONCLUSÃO}

Este estudo descreve a freqüência de fatores de risco tradicionais para doença arterial coronariana em uma amostra significativa de pacientes com LES, sendo o trabalho publicado na literatura nacional com maior casuística. Alguns dos fatores mais freqüentes são modificáveis, como a obesidade, ou controláveis, como a HAS e a dislipidemia. Esclarecimento, tanto para pacientes quanto para médicos envolvidos no tratamento do LES, sobre a importância da aterosclerose como fator de morbidade e mortalidade nesses doentes e a presença desses fatores de risco devem ser preocupações constantes durante o acompanhamento da doença. O impacto da identificação e do tratamento de fatores de risco em pacientes com lúpus para prevenção de eventos cardiovasculares deve ser objeto de novos estudos.

Declaramos a inexistência de conflitos de interesse. 


\section{REFERÊNCIAS}

1. Barr SG, Zonana-Nacach A, Magder LS, Petri M: Patterns of disease activity in systemic lupus erythematosus. Arthritis Rheum 42:2682-8, 1999.

2. Bessant R, Hingorani A, Patel L, MacGregor A, Isemberg DA, Rahman A: Risk of coronary heart disease and stroke in a large British cohort of patients with systemic lupus erythematosus. Rheumatology 43:924-9, 2004.

3. Esdaile JM, Abrahamowicz M, Grodzicky T, Li Y, Panaritis C, du Berger R: Traditional Framingham risk factors fail to fully account for accelerated atherosclerosis in systemic lupus erythematosus. Arthritis Rheum 44:2331-7, 2001.

4. Manzi S, Meilahn EN, Rairie JE, et al.: Age-specific incidence rates of myocardial infarction and angina in women with systemic lupus erythematosus: comparison with the Framingham study. Am J Epidemiol 145:408-15, 1997.

5. Aranow C, Ginzler EM: Epidemiology of cardiovascular disease in systemic lupus erythematosus. Lupus 9:166-9, 2000.

6. Petri M, Perez-Gutthann S, Spence D, Hochberg MC: Risk factors for coronary artery disease in patients with systemic lupus erythematosus. Am J Med 93:513-9, 1992.

7. Gladman DD, Urowitz MB: Morbidity in systemic lupus erythematosus. J Rheumatol 14:223-6, 1987.

8. Rahman P, Gladman DD, Urowitz MB, Yuen K, Hallett D, Bruce IN: The cholesterol lowering effect of antimalarials drugs is enhanced in patients with lupus taking corticosteroid drugs. J Rheumatol 26:325-30, 1999.

9. Souza AWS, Hatta FS, Miranda JF, Sato EI: Atherosclerotic plaque in carotid arteries in systemic lupus erythematosus frequency and associated risk factors. São Paulo Medical Journal 123:137-42, 2005.

10. Bruce IN, Urowitz MB, Gladman DD, Ibanes D, Steiner G: Risk factors for coronary heart disease in women with systemic lupus erythematosus: the Toronto risk factor study. Arthritis Rheum 48:3159-67, 2003.

11. Rahman P, Aguero S, Gladman DD, Hallett D, Urowitz MB: Vascular events in hypertensive patients with systemic lupus erythematosus. Lupus 9:672-5, 2000.

12. Toloza SMA, Uribe AG, McGwin G-Jr, et al.: Systemic lupus erythematosus in a multiethinic US cohort (LUMINA): XXIII. Baseline predictors of vascular events. Arthritis Rheum 50: 3947-57, 2004.

13. Petri M, Spence D, Bone LR, Hochberg MC: Coronary artery disease risk factors in the Johns Hopkins Lupus Cohort: prevalence, recognition by patients, and preventive practices. Medicine 71:291-302, 1992.

14. Costenbader KH, Wright E, Liang MH, Karlson EW: Cardiac risk factor awareness and management in patients with systemic lupus erythematosus. Arthritis Rheum 51:983-8, 2004.

15. Urowitz MB, Gladman DD, Fortin PR, et al.: Systemic Lupus International Collaborating Clinics (SLICC) inception cohort registry to study risk factors for atherosclerosis: accumulation of atherosclerotic risk factors over 3 years. Arthritis and Rheumatism 54:S521, 2006.

16. Expert panel on detection, evaluation, and treatment of high blood cholesterol in adults - Executive Summary of the third report of the National Cholesterol Education Program (NCEP) (Adult Treatment Panel III). JAMA 285:2486-97, 2001.

17. Wajed J, Ahmad Y, Durrington PN, Bruce IN: Prevention of cardiovascular disease in systemic lupus erythematosus proposed guidelines for risk factor management. Rheumatology 43:7-12, 2004.

18. Hochberg MC: Updating the American College of Rheumatology revised criteria for the classification of the systemic lupus erythematosus (letter). Arthritis Rheum 40:1725, 1997.

19. Tam EM, Cohen AS, Fries JF: The 1982 revised criteria for the classification of systemic lupus erythematosus. Arthritis Rheum 25:1271, 1982 .

20. Gladman DD, Urowitz MD: Systemic Lupus Erythematosus Clinical Features. In: Klippel JH, Dieppe PA (eds): Rheumatology. $2^{\text {a }}$ ed. Londres, Fiona Foley, 1998.

21. Wilson WA, Gharavi AE, Koike T, et al.: International consensus statement on preliminary classification criteria for definite antiohospholipid syndrome-Report of an international workshop. Arthritis Rheum 42:1309-11, 1999.

22. Gladman DD, Ginzler EM, Goldsmith C, et al.: The Development and initial validation of the Systemic Lupus International Collaborating Clinics/American College of Rheumatolgy damage index for systemic lupus erythematosus. Arthritis Rheum 39:363-9, 1996.

23. European Society of Cardiology, European Atherosclerosis Society, European Society of Hypertension, International Society of Behavioral Medicine, European Society of General Practice/ Family Medicine, European Heart Network: Prevention of coronary heart disease in clinical practice: recommendations of the second Joint Task Force of European and the other Societies on coronary prevention. Eur Heart J 19:1434-503, 1998.

24. Diagnosis and classification of Diabetes Mellitus - American Diabetes Association. Diabetes Care 27:S5-S10, 2004

25. O'Leary DH, Polak JF, Kronmal RA, et al.: Distribution and correlates of sonographically detected carotid artery disease in the Cardiovascular Health Study. Stroke 23:1752-60, 1992.

26. Theodoridou A, Bento L, D'Cruz DP, Khamashta MA, Hughes GRV: Prevalence and association of an abnormal ankle-brachial in systemic lupus erythematosus: a pilot study. Ann Rheum Dis 62:1199-203, 2003.

27. Prevenção clínica de doenças cardiovascular, cerebrovascular e renal - Manual prático para profissionais do Sistema Único de Saúde Caderno de atenção básica - Estimativa de risco global e prevenção. Ministério da Saúde - Secretaria de Atenção à Saúde - Departamento de Atenção Especializada/Coordenação Geral de Alta Complexidade - Coordenação de Hipertensão e Diabetes, ed., 2006.

28. Wilson PWF, D’Agostino RB, Levy D, Belanger AM, Silbershatz H, Kannel WB: Prediction of coronary heart disease using risk factor categories. Circulation 97:1837-47, 1998.

29. Urowitz MB, Gladman DD, Ibanez D, et al.: Systemic Lupus International Collaborating Clinics (SLICC) inception cohort registry to study risk factors for atherosclerosis: report on the first 852 patients. Arthritis Rheum 54:S281, 2006.

30. Sella EMC, Sato EI: Avaliação de fatores de risco coronário e dor torácica em lúpus eritematoso sistêmico. Rev Bras Reumatol 42:160-8, 2002. 
31. Barreto SM, Passos VMA, Cardoso ARA, Lima-Costa MF Quantificando o risco de doença coronariana na comunidade de Bambuí. Arq Bras Cardiol 81:549-55, 2003.

32. Avalos I, Chung CP, Oeser A, et al.: Decreased insulin sensitivity in systemic lupus erythematosus is associated with inflammation and cardiovascular risk factors. Arthritis Rheum 54:S271, 2006.

33. Toloza S, Chandran V, Gladman DD, Ibanes D, Urowitz M: Metabolic Syndrome in SLE: increased prevalence and associations with disease manifestations. Arthritis Rheum 54:S434, 2006.
34. Urowitz MB, Gladman DD, Fortin PR, et al.: Systemic Lupus International Collaborating Clinics (SLICC) inception cohort registry to study risk factors for atherosclerosis: metabolic syndrome, prevalence and associated factors. Arthritis Rheum 54:S282, 2006.

35. Borba EF, Bonfá E: Dyslipoproteinemias in systemic lupus erythematosus: influence of disease, activity, and anticardiolipins antibodies. Lupus 6:533-9, 1997.

36. Bruce IN, Urowitz MB, Gladman DD, Hallett DC: Natural history of hypercholesterolemia in systemic lupus erythematosus. J Rheumatol 26:2137-43, 1999. 